\title{
Factors influencing the temperature sensitivity of PMMA based optical fiber Bragg gratings
}

\author{
Wei Zhang* and David J. Webb \\ Aston Institute of Photonic Technologies, Aston University, Birmingham, B4 7ET, UK
}

\begin{abstract}
The Bragg wavelength of a PMMA based fiber grating is determined by the effective core index and the grating pitch, which, in temperature sensing, depend on the thermo-optic and thermal expansion coefficients of PMMA. These two coefficients are a function of surrounding temperature and humidity. Amorphous polymers including PMMA exhibit a certain degree of anisotropic thermal expansion. The anisotropic nature of expansion mainly depends on the polymer processing history. The expansion coefficient is believed to be lower in the direction of the molecular orientation than in the direction perpendicular to the draw direction. Such anisotropic behavior of polymers can be expected in drawn PMMA based optical fiber, and will lead to a reduced thermal expansion coefficient and larger temperature sensitivity than would be the case were the fiber to be isotropic. Extensive work has been carried out to identify these factors. The temperature responses of gratings have been measured at different relative humidity. Gratings fabricated on annealed and non-annealed PMMA optical fibers are used to compare the sensitivity performance as annealing is considered to be able to mitigate the anisotropic effect in PMMA optical fiber. Furthermore an experiment has been designed to eliminate the thermal expansion contribution to the grating wavelength change, leading to increased temperature sensitivity and improved response linearity.
\end{abstract}

Keywords: PMMA based optical fiber, fiber Bragg grating, anisotropic expansion, thermo-optic effect

\section{INTRODUCTION}

Fiber Bragg grating (FBG) sensors inscribed in silica optical fiber have become an increasingly mature sensing technology. More recently, FBG sensors have been inscribed into poly(methyl methacrylate) (PMMA) based plastic optical fiber in both step-index and microstructured geometries. The physical and chemical properties of polymeric materials are rather different to silica and may offer advantages in certain situations. Polymer optical fiber Bragg gratings (POFBGs) are particularly appealing in medical devices, as polymers are biologically compatible with human tissues and already routinely put in the human body in many applications.

One of the interesting features of polymer fiber gratings is the negative refractive index change with temperature increase. It offers a well-conditioned behavior useful in overcoming the cross sensitivity issues existing in silica fiber gratings [1][2]. There have been many reports of polymer fiber grating sensing applications involving temperature. However, the reported results on the temperature response of PMMA based fiber gratings are quite varying. In the 1550nm region PMMA fiber gratings show a temperature sensitivity from $-10 \mathrm{pm} /{ }^{\circ} \mathrm{C}[3],-55 \mathrm{pm} /{ }^{\circ} \mathrm{C}[4]$ to $-360 \mathrm{pm} /{ }^{\circ} \mathrm{C}$ [5]. This causes great uncertainty in POFBG's performance and applicability. The principle of POFBG temperature sensing is well known. However, the mechanism of the PMMA optical fiber's response to temperature is far more complex than that has so far been reported.

\section{PRINCIPLE OF POFBG TEMPERATURE SENSING}

\subsection{Characteristic wavelength of POFBG}

The Bragg wavelength of an unstrained fiber grating depends on the effective core index, $n_{\text {eff }}$, and the grating pitch, $\Lambda$, both of which depend on the temperature $T$ and the water content $w$ in the case of a POFBG. For PMMA based fiber grating the Bragg wavelength can then be expressed as

*w.zhang@aston.ac.uk

\footnotetext{
Micro-structured and Specialty Optical Fibres III, edited by Kyriacos Kalli,

Alexis Mendez, Proc. of SPIE Vol. 9128, 91280M · ( 2014 SPIE

CCC code: $0277-786 X / 14 / \$ 18 \cdot$ doi: $10.1117 / 12.2054210$
} 


$$
\lambda_{\text {Bragg }}=2 n_{\text {eff }}(T, w) \Lambda(T, w)
$$

For constant relative humidity $(\mathrm{RH})$ the Bragg wavelength change as a result of varying temperature can be expressed as

$$
\Delta \lambda_{B}=\lambda_{B 0}(\alpha+\xi / n) \Delta T
$$

where $\lambda_{B 0}$ is initial Bragg wavelength, $\alpha$, the thermal expansion coefficient (TEC), $\xi$, the thermo-optic coefficient (TOC). It is well known now that the thermo-optic coefficient of PMMA is negative and its contribution to the wavelength change of POFBG is larger than that that of thermal expansion of PMMA. Therefore the wavelength change of a PMMA based POFBG exhibits a blue shift against temperature increase.

\subsection{TEC and TOC of PMMA}

In the case of PMMA optical fibers, the thermal expansion coefficient $\alpha=L^{-1} \partial L / \partial T$ is a function of temperature and water content in the fiber $[6,7]$; we may define the TEC as

$$
\alpha=\alpha_{0}+\alpha_{1} T+\alpha_{2} w+\alpha_{3} T w
$$

In analogy, we assume that the thermo-optic coefficient follows a similar relation,

$$
\xi=\xi_{0}+\xi_{1} T+\xi_{2} w+\xi_{3} T w
$$

The dependence of the optical properties of PMMA on humidity and temperature has been extensively investigated by different authors [6-8]. Although the reported works were carried out for bulk PMMAs in different operating wavelength regions the results still have generality and can be used to predict the performance of PMMA optical fibers.

The thermal expansion of PMMA increases with increasing temperature [6][7]. The effects of moisture on the thermal expansion of compression-moulded PMMA were investigated by dilatometry in [8]. Moisture conditioning at high relative humidity, immersion in water, or vacuum drying were used to prepare PMMA specimens at different moisture contents [9]. Least-squares fitting was used to determine the constant and linear CTE terms as a function of percent weight gain to provide an expression for the CTE of PMMA against weight gain and temperature, as follows

$$
\alpha(T, w)=(59.3+4.26 w+0.222 T+0.139 w T) \times 10^{-6}
$$

where $w$ is in \% weight gain. Here the maximum $w$ is 2.02 , corresponding to $100 \%$ relative humidity.

The TOC of PMMA can be obtained based on the results retrieved from [6]. By using a least squares fit we can obtain the thermo optic coefficients of PMMA as

$$
\xi(T, H)=(-60.388-20.052 H-1.953 T-0.477 H T) \times 10^{-6}
$$

where $H$ is the relative humidity. The above equation is regressed on the data measured in the range of 20 to $90{ }^{\circ} \mathrm{C}$ and 20 to $90 \% \mathrm{RH}$.

\subsection{Performance of PMMA based optical fibers}

Bragg gratings can be written into pure PMMA optical fibers by using UV illumination which induces polymerization of unreacted monomer that contributes to the refractive index increase. The photosensitivity of pure PMMA is low and varies along the fiber length [10]. To improve its performance, many doped-core PMMA based optical fibers have been proposed. A lot of reported polymer fiber gratings are written into doped PMMA based optical fibers for temperature sensing application. Many of them involve step indexed fiber. Since additional dopants or copolymers are required to control the core index of step index fibers, the performance of PMMA based fiber gratings may vary with the types of fiber used. The detailed composition of dopant and copolymer used in a specific PMMA based optical fiber is often not known and neither is its dependence on temperature and humidity. However, since the fiber cladding, which is usually made of PMMA, takes the largest portion of the fiber's volume or weight, we assume the fiber performance can be dominated and approximated by that of pure PMMA.

\section{MEASUREMENT OF POFBG TEMPERATURE RESPONSES}

Experiments were carried out to inspect the responses of a POFBG to temperature. POFBGs were fabricated by attaching a several centimeter length of step index POF to a single-mode silica fiber down-lead using UV curable glue. The PMMA based POF contained a $10 \mathrm{~mm}$ long FBG, fabricated by illuminating from above a phase mask placed on top of 
the POF using $325 \mathrm{~nm}$ UV light from a HeCd laser. A broadband light source with a total output optical power of $10 \mathrm{~mW}$ was used, launching light via a fiber circulator into the POFBG under investigation. In the experiments the POFBGs were placed inside an environmental chamber (Sanyo Gallenkamp) so as to operate at the desired temperature and humidity. The reflection of the POF grating was monitored by using an IBSEN I-MON 400 wavelength interrogation system. The experimental arrangement is shown in Fig. 1.

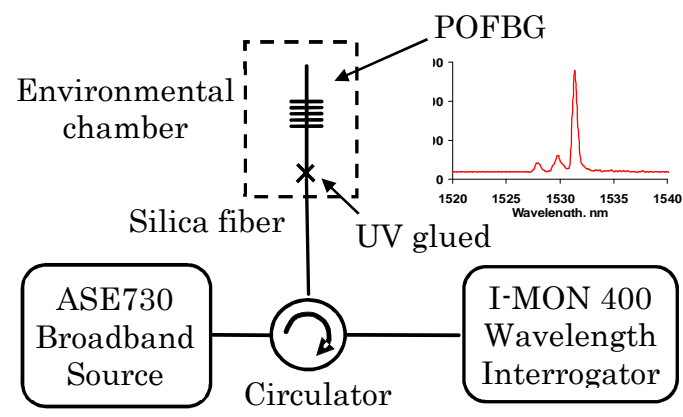

Fig. 1 Experimental arrangement for investigating POFBG responses over temperature and humidity

\section{RESULTS AND DISCUSSIONS}

To investigate the temperature response of the POFBG sensors, the POFBG was placed in the chamber under varying temperatures while relative humidity was fixed. The temperature response of a POFBG sensor is illustrated in Fig. 2 while the environmental chamber was set at a relative humidity of $55 \%$.

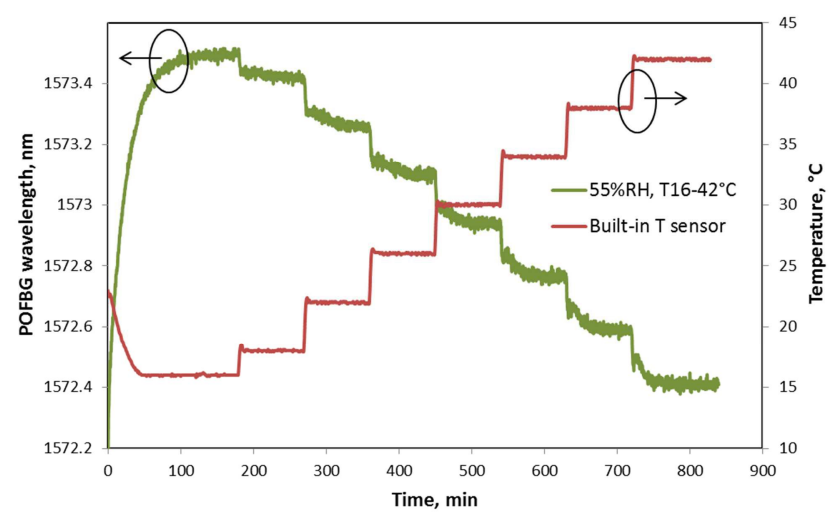

Fig. 2 A recorded temperature response of POFBG

The temperature response of a POFBG was measured at different humidity levels. The thermal expansion and thermooptic performance of dopants and copolymers used in most step index PMMA based fibers have not been reported; consequently, we use the parameters of PMMA as an approximation in estimating POFBG temperature sensitivity. Some measured results are plotted in Fig. 3 in comparison with calculation based on PMMA's coefficients of thermal expansion and thermo-optic effect defined by (5) and (6). It can be seen that the measured result shows a good linearity and a sensitivity of $-42 \mathrm{pm} /{ }^{\circ} \mathrm{C}$ at $55 \% \mathrm{RH}$ and $-51 \mathrm{pm} /{ }^{\circ} \mathrm{C}$ at $85 \% \mathrm{RH}$. However, it is not in agreement with the calculation. This indicates that the POFBG performance cannot be predicted by simply using (2) and PMMA parameters, defined by (5) and (6). The factors affecting POFBG temperature response need to be examined. 


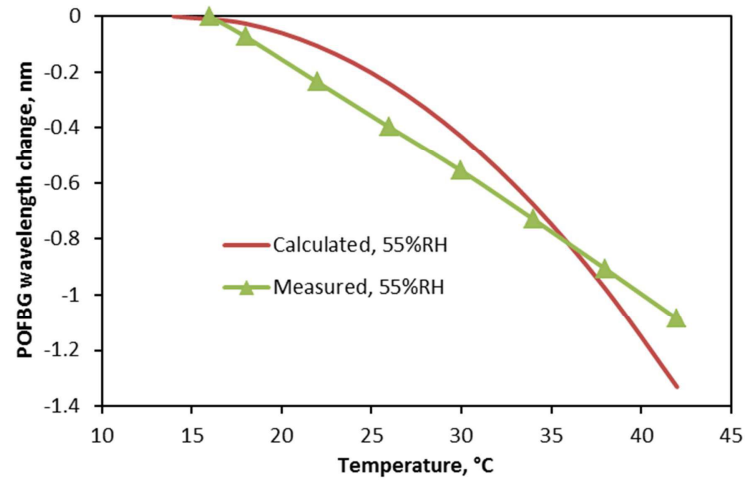

(a)

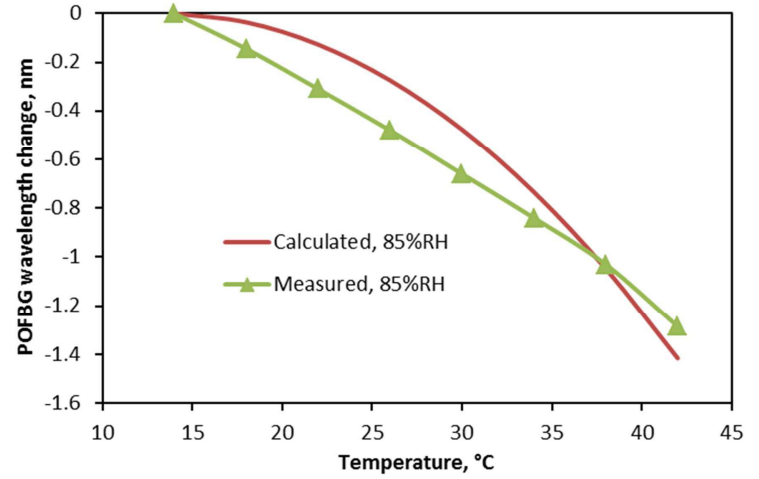

(b)

Fig. 3 Measured and calculated POFBG responses based on PMMA's performance (a) at 55\% RH, (b) at $85 \%$ RH

\subsection{POFBG annealing effect}

Polymer optical fiber is pulled under tension, resulting in an axial orientation of the polymer chains and residual strain in the fiber. However, at a certain temperature, the chains are thought to relax from the axial orientation causing shrinkage of the fiber. In this case POFBG wavelength decreases rapidly with increasing temperature and introduces a permanent wavelength shift [11].

Figure 4 shows the temperature responses of a POFBG. The initial wavelength of the POFBG was $1536.86 \mathrm{~nm}$ at $14^{\circ} \mathrm{C}$ and $85 \% \mathrm{RH}$. Then the temperature increased with steps of $4^{\circ} \mathrm{C}$ up to $42^{\circ} \mathrm{C}$ while the relative humidity was held at $85 \%$. One can see that before $38^{\circ} \mathrm{C}$ the POFBG wavelength always reaches a stable value; after $38^{\circ} \mathrm{C}$ the POFBG wavelength keeps dropping. In the end of the experiment the POFBG wavelength already fell below $1531 \mathrm{~nm}$ and never went back to the original value. The same POFBG was used to repeat the experiment. The initial wavelength at $14^{\circ} \mathrm{C}$ and $85 \% \mathrm{RH}$ now was $1530.55 \mathrm{~nm}$ - a permanent wavelength shift of $-6.3 \mathrm{~nm}$. From Fig. 5 one can see that the POFBG wavelength drops slower than it did in the first experiment. After $38^{\circ} \mathrm{C}$ the POFBG wavelength still keeps dropping.

This effect has been reported previously to be a function of the drawing tension [11]. The temperature at which this effect starts is dependent on the thermal history of the grating and could be related to the $\beta$ relaxation in PMMA when the side chains of the polymer begin to move. According to [12] the $\beta$ relaxation temperature for bulky $\mathrm{PMMA}$ is $40^{\circ} \mathrm{C}$ and can be as low as $34^{\circ} \mathrm{C}$ in some cast PMMA films. This phenomenon has been reported in [13] and a solution was also given to eliminate this effect by pre-annealing PMMA based optical fiber. A POFBG was written into the POF that was pre-annealed in an oven at $80^{\circ} \mathrm{C}$ for 7 hours. The experiment was repeated using this annealed POFBG and the measured wavelength change of the annealed POFBG against temperature is plotted in Fig. 5, in comparison with those of the un-annealed POFBG. The response of the annealed POFBG exhibits smaller sensitivity but better linearity. More important is that this POFBG experiences no permanent wavelength shift and its response is repeatable.

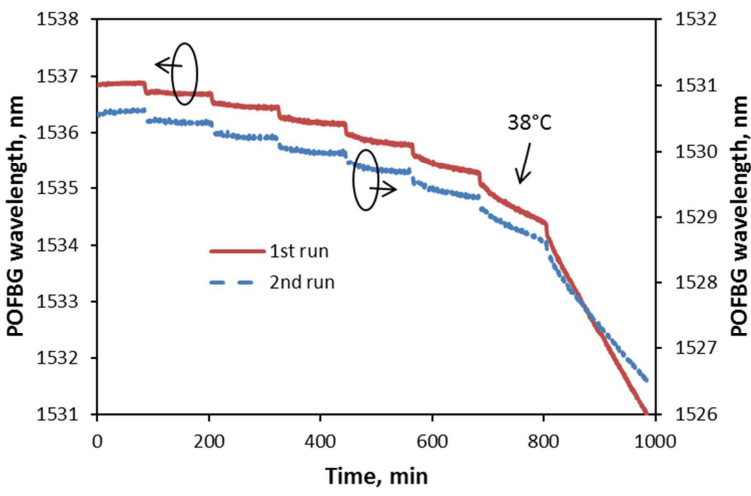

Fig. 4 Un-annealed POFBG temperature responses

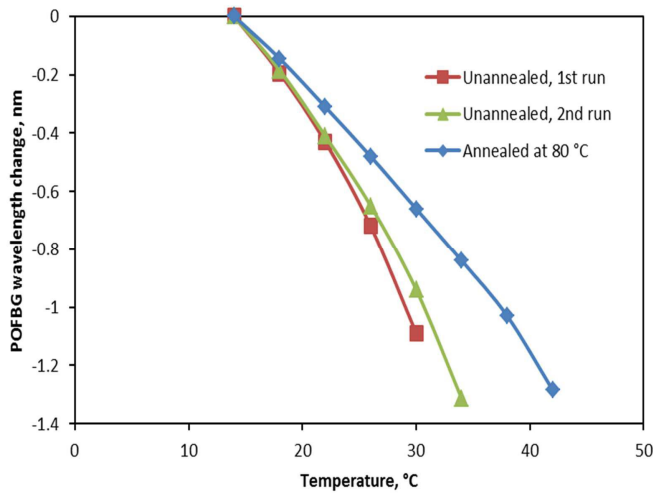

Fig. 5 Comparison of POFBG wavelength responses 


\subsection{Anisotropic thermal expansion of PMMA based optical fiber}

The thermal expansion coefficient of PMMA has been measured by using the optical interference method [7] or estimated from the volume change by using a dilatometer [8]. In both cases PMMA was assumed isotropic so the linear thermal expansion coefficient is a third of the volume change caused by thermal expansion.

However, amorphous polymers including PMMA exhibit a known degree of anisotropic thermal expansion. The anisotropic nature of expansion mainly depends on the polymer processing history and is typical of solid drawn polymers and stretched elastomers [14]. Furthermore, the expansion coefficient is believed to be lower in the direction of the orientation than in the direction perpendicular. In some cases with large draw ratio the thermal expansion in the drawing direction can even be negative. POFs have extremely large draw ratio and may fall in this category.

In fact the thermal performance of the un-annealed POFBG studied in this work implies that a negative thermal expansion exists in the raw POFs: the permanent wavelength downshift arises from fiber shrinkage. Normal thermal expansion of PMMA is positive and it counteracts the contribution of the thermo-optic effect to the POFBG wavelength response. Therefore the annealed POFBG exhibits smaller temperature sensitivity than that of the un-annealed device (see Fig. 5). A reduced thermal expansion in the fiber direction may explain the better linearity in the measured response than in the calculation (see Fig. 3), as in fact the calculated response shows better linearity if the thermal expansion contribution is minimized. We have noticed that this anisotropic effect also exists in the swelling of PMMA baser optical fiber due to water absortption which consequently reduces the humidity sensitivity of a POFBG [15].

For uniaxially oriented polymers, one simple and commonly used measure of orientation is the birefringence, $\Delta n$, which is the difference of refractive index for light polarized in the draw direction and light polarized perpendicular to the draw direction [16]. An orientation function which is proportional to $\Delta n$, can be expressed as, $f=1 / 2\left(3 \overline{\cos ^{2} \theta}-1\right)$, where $\theta$ is the angle between the draw direction and the axis of the intrinsic unit. The bar denotes the average over the aggregate. Due to the large draw ratio in PMMA based optical fiber (>6000) [17], $\theta$ is very small and $f$ close to one. Furthermore, the orientation function can be expressed by the thermal expansion coefficients,

$$
f=\frac{2}{3} \frac{\alpha_{\perp}-\alpha_{\|}}{\alpha_{i s o}}
$$

where $\alpha_{/ /}, \alpha_{\perp}$ and $\alpha_{i s o}$, are the thermal expansion coefficients in the draw direction, perpendicular to the draw direction and for isotropic polymer. Since $f \approx 1$ this gives $3 \alpha_{i s o}=2\left(\alpha_{\perp^{-}} \alpha_{/ 6}\right)$. This means that there exists either significantly increased $\alpha_{\perp}$ or significantly reduced $\alpha_{/ /}$, or both. In highly oriented polymer it is increased $\alpha_{\perp}$ and negative $\alpha_{/ 6}$ [18].

\subsection{Thermo-optic effect of PMMA based optical fiber}

Since the polymer chains in POF are highly oriented in the axial direction, such anisotropic thermal expansion can also even be expected in the annealed POFs [17], which is implied by the better linearity in the measured temperature responses of POFBGs than that in those calculated. Removal of the thermal expansion contribution to the POFBG wavelength response will lead to a good linearity and larger sensitivity.

An experiment was designed to demonstrate this. The two ends of a POFBG were clamped to two mounting brackets. One of the brackets was fixed while the other was mounted on a translation stage, which was used to apply strain to the POFBG. The clamped POFBG was then placed in the environmental chamber to test the temperature sensitivity at 55\% RH. Since the ends of the POFBG were clamped the length of the PMMA optical fiber between the two clamping points does not vary with POF expansion (given that the applied strain was larger than any temperature induced fiber expansion). In this case the POFBG temperature sensitivity only relies on the thermo-optic effect of the fiber. The measured POFBG response is shown in Fig. 6 where a strain of $3500 \mu \varepsilon$ had been applied and temperature varied from $18^{\circ} \mathrm{C}$ to $42^{\circ} \mathrm{C}$. The POFBG wavelength against temperature was plotted in the inset in Fig. 6, indicating a good linearity and a sensitivity of $-79 \mathrm{pm} /{ }^{\circ} \mathrm{C}$. Compared to the unstrained POFBG $\left(-42 \mathrm{pm} /{ }^{\circ} \mathrm{C}\right.$ as shown in Fig. 3), the pre-strained POFBG exhibits a much larger temperature sensitivity. 


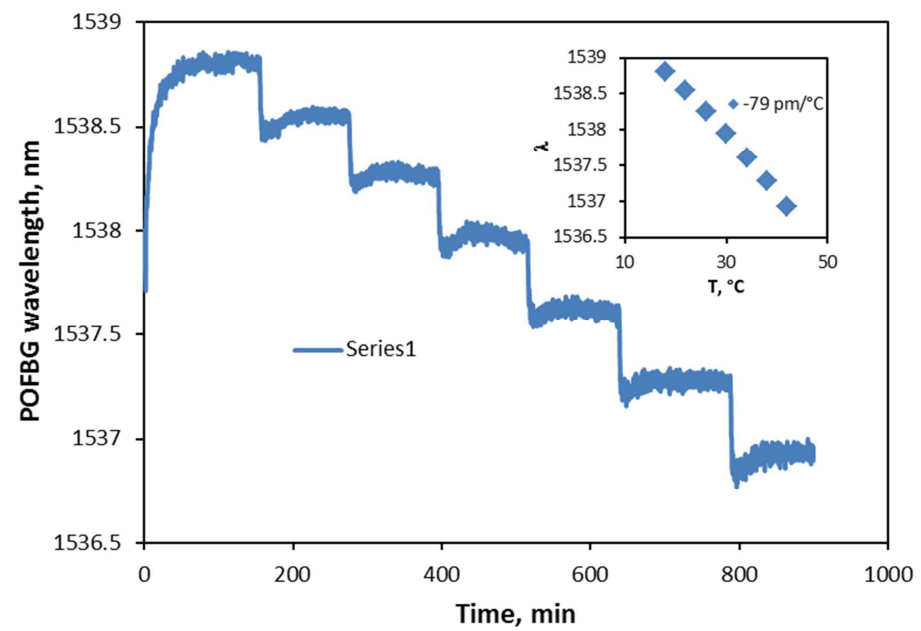

Fig. 6 A pre-strained POFBG wavelength response. Inset: POFBG wavelength vs. temperature.

In estimating the temperature sensitivity of a pre-strained POFBG, the values of TEC and TOC in the selected temperature range and humidity can be obtained from [6,9]. However, the magnitude of the calculated temperature sensitivity of a pre-strained POFBG is much larger than achieved in the experiment, even though the varying values of TEC and TOC have been considered. This disagreement may be explained by a much reduced thermal expansion in prestrained POFBG. According to Prod'homme theory [19] the thermo-optic coefficients of optical polymers depends on electronic polarizability and density,

$$
\zeta=\frac{d n}{d T}=\frac{\left(n^{2}-1\right)\left(n^{2}+2\right)}{6 n}(\phi-\beta)
$$

where $\phi$ is the temperature coefficient of the electronic polarizability and $\beta$ the volume expansion coefficient. If the optical fiber cannot expand and contract freely its thermal expansion is restrained and its density change is reduced [18]. In a pre-strained POFBG the linear expansion is greatly restricted by the strain applied, leading to a reduced thermooptic coefficient and consequently a smaller contribution of thermo-optic refractive index change to the POFBG wavelength response is obtained.

\section{ACKNOWLEDGEMENT}

The research leading to these results has received funding from the European Union Seventh Framework Programme (FP7/2007-2013) under grant agreement no. 314032.

\section{REFERENCES}

[1] Liu, H. B., Liu, H. Y., Peng, G. D., and Chu, P. L.," "Strain and temperature sensor using a combination of polymer and silica fibre bragg gratings," Opt. Commun. 219, 139-142 (2003)

[2] Xu, M. G., Archambault, J.-L., Reekie, L. and Dakin, J. P., "Discrimination between strain and temperature effects using dual-wavelength fiber grating sensors," Electron. Lett. 30(13), 1085-1087 (1994).

[3] Harbach, G. N., Limberger, Hans G., and Salathé, René P., "Influence of Humidity and Temperature on Polymer Optical Fiber Bragg Gratings," Proc. Bragg Gratings, Photosensitivity, and Poling in Glass Waveguides, BTuB2, (2010).

[4] Zhang, C., Zhang, W., Webb, D. J., Peng, G.-D., "Optical fiber temperature and humidity sensor," Electron. Lett., 46(9) pp. 643 (2010).

[5] Liu, H. Y., Peng, G. D., and Chu, P. L., "Thermal tuning of polymer optical fiber bragg gratings," IEEE Photon.Technol. Lett. 13, 824 (2001).

[6] Watanabe, T., Ooba, N., Hida, Y., and Hikita, M., "Influence of humidity on refractive index of polymers for optical waveguide and its temperature dependence", Appl. Phys. Lett., 72(13), 1533 (1998) 
[7] Waxler, R. M., Horowitz,D., and Feldman, A., "Optical and physical parameters of Plexiglas 55 and Lexan," Appl. Opt. 18(1), 101 (1979)

[8] Startsev, O. V., Rudnev, V. P., "Reversible moisture effects in the climatic ageing of organic glass," Polymer Degradation and Stability 39, 373-379 (1993)

[9] W. D. Drotning, E. P. Roth, "Effects of moisture on the thermal expansion of poly( methyl metacrylate )," Journal of Materials Science 24, 3137-3140 (1989)

[10] Harbach, N. G., "Fiber Bragg gratings in polymer optical fibers," Ph.D. dissertation, Ecole Polytechnique Fédérale de Lausanne, (2008).

[11] Kuzyk, M. G., [Polymer Fiber Optics: Materials, Physics, and Applications], CRC Press, (2007).

[12] Wypych, G., [Handbook of solvents], ChemTec Publishing, (2001)

[13] Carroll1, K. E., Zhang, C., Webb, D. J., Kalli, K., Argyros, A., Large, M. C. J., "Thermal response of Bragg gratings in PMMA microstructured optical fibers," Opt. Express 15(14), 8844-8850 (2007).

[14] Fakirov, S., [Oriented Polymer Materials], WILEY-VCH, (2002).

[15]Zhang, W., and Webb, D. J., "Humidity responsivity of polymer optical fiber Bragg grating sensors," to appear in Opt. Lett.

[16] Wang, L. -H., Choy, C. L., and Porter, R. S., "Thermal Expansion of Oriented Poly(methy1Methacrylate)", Journal of Polymer Science 21, 657-665 (1983).

[17] Ji, P., Li, A. D. Q., and Peng, G. -D., "Transverse birefringence in polymer optical fibre introduced in drawing process," Proceedings of SPIE Vol. 5212, 108-116 (2003).

[18] Diemeer M. B. J., "Polymeric thermo-optic space switches for optical communications," Optical Materials 9 , 192-200 (1998).

[19] Prod'Homme, L., "A new approach to the thermal change in the refractive index of glasses," Phys. and Chem. of Glasses 1, 119-122 (1960) 